INPLASY

PROTOCOL

To cite: Li et al. A Bayesian network meta-analysis of acupuncture to treat vascular dementia in animal models. Inplasy protocol 2021110036. doi:

10.37766/inplasy2021.11.0036

Received: 11 November 2021

Published: 11 November 2021

Corresponding author:

Guangyao Li

425293675@qq.com

Author Affiliation:

Acupuncture and Rehabilitation

Clinical Medical College,

Guangzhou University of Chinese

Medicine.

Support: Natural Science

Foundation.

Review Stage at time of this submission: Risk of bias assessment.

Conflicts of interest:

None declared.

\section{A Bayesian network meta-analysis of acupuncture to treat vascular dementia in animal models}

Review question / Objective: Whether acupuncture can improve cognition in vascular dementia animals compared to any other intervention. Population (P): vascular dementia animal models. Intervention (I): acupuncture, eletroacupuncture, warm needling, scalp-acupuncture. Comparison (C): Sham /placebo acupuncture, Conventional medicine, Western medicine,sham operation, and surgical model. Outcomes (0): Water maze (escape latency, amount of time spent in the original platform).

Condition being studied: Relying on the research background of Guangzhou University of Chinese Medicine, we formed a research team with master's degree and doctor's degree to study evidence-based medicine. According to the hot spots of scientific research, the literature on acupuncture treatment of vascular dementia was retrieved and screened, the included literature was evaluated, and the data were extracted. Multivariate analysis of the extracted data was conducted with The Bayesian method, and reliable conclusions were obtained. We searched and screened the literature, evaluated the included literature, and extracted the data.

INPLASY registration number: This protocol was registered with the International Platform of Registered Systematic Review and Meta-Analysis Protocols (INPLASY) on 11 November 2021 and was last updated on 11 November 2021 (registration number INPLASY2021110036).

\section{INTRODUCTION}

Review question / Objective: Whether acupuncture can improve cognition in vascular dementia animals compared to any other intervention. Population (P): vascular dementia animal models.
Intervention (I): acupuncture, eletroacupuncture, warm needling, scalpacupuncture. Comparison (C): Sham / placebo acupuncture, Conventional medicine, Western medicine,sham operation, and surgical model. Outcomes 
(0): Water maze (escape latency, amount of time spent in the original platform).

Rationale: Vascular dementia is the second largest type of dementia, and there is no specific drug treatment for it. Animal experiments carried out in recent years have proved that acupuncture play an important role in the treatment of vascular dementia, but its physiological mechanism has not been fully elucidated. The purpose of this systematic review is to analyze the effect of acupuncture on improving cognition in animal models of vascular dementia, further explore the law of acupoint use, summarize the physiological mechanism of action, and comprehensively evaluate the efficacy differences of acupuncture, sham acupuncture, western medicine, and model group in animal models.

Condition being studied: Relying on the research background of Guangzhou University of Chinese Medicine, we formed a research team with master's degree and doctor's degree to study evidence-based medicine. According to the hot spots of scientific research, the literature on acupuncture treatment of vascular dementia was retrieved and screened, the included literature was evaluated, and the data were extracted. Multivariate analysis of the extracted data was conducted with The Bayesian method, and reliable conclusions were obtained.We searched and screened the literature, evaluated the included literature, and extracted the data.

\section{METHODS}

Search strategy: Our search time is limited to the establishment of the database until October 2021. The search terms are : acupuncture, acupuncture, electroacupuncture, warm cupuncture, scalp acupuncture, acupoints, vascular dementia, infarction dementia, vascular cognitive impairment,animal. Each search term is used alone or in combination.We started by searching four authoritative international journal databases in English: PubMed, Medline, Embase and Web of
Science. Then search CNKI, Wanfang and VIP databases in Chinese.

Participant or population: Animal experimental studies.

Intervention: Acupuncture, sham acupuncture, Western medicine.

Comparator: Surgery model, sham operation.

Study designs to be included: Inclusion criteria: This study only included animal experimental studies with a control design for acupuncture treatment of vascular dementia, and the control group could be one or more. Exclusion criteria: Interventions no acupuncture study, no control study, Study for human body.

Eligibility criteria: The animal species included in this study were not limited, and the modeling methods included bilateral common carotid artery occlusion, fourvessel occlusion, middle cerebral artery occlusion, and Embolic occlusion.

Information sources: We started by searching four authoritative international journal databases in English: PubMed, Medline, Embase and Web of Science. Then search CNKI, Wanfang and VIP databases in Chinese.

Main outcome(s): Morris Water Maze Test (escape latency, amount of time spent in the original platform).

Additional outcome(s): Frequency of use of acupuncture points, Success rate of various moulding methods.

Quality assessment / Risk of bias analysis: The two researchers independently used the SYRCLE' $s$ risk of bias tool to evaluate the included literature. If they encountered differences, they would be solved through discussions with the third author. SYRCLE, s risk of bias include: (1) Was the allocation sequence adequately generated and applied? (2) Were the groups similar at baseline or were they adjusted for 
confounders in the analysis? (3) Was the allocation adequately concealed? (4) Were the animals randomly housed during the experiment? (5) Were the caregivers and/or investigators blinded from knowledge which intervention each animal received during the experiment? (6) Were animals selected at random for outcome assessment? (7) Was the outcome assessor blinded? (8) Were incomplete outcome data adequately addressed? (9) Are reports of the study free of selective outcome reporting? (10) Was the study apparently free of other problems that could result in high risk of bias?

Strategy of data synthesis: Network metaanalysis is an extension of direct metaanalysis, which uses indirect comparison techniques to comprehensively evaluate and rank all interventions in the same body of evidence. We use the analysis software ADDIS 16.8 based on Bayesian principle to make direct comparison and indirect comparison analysis. Firstly, we make direct comparison analysis, check heterogeneity through $I$, and integrate the results of direct comparison and corresponding forest map description. Secondly, the network meta-analysis was carried out under the Bayesian framework based on Markov chain Monte Carlo, the results of the included interventions were sorted, and the network diagram was drawn using the network plot command of stata.

Subgroup analysis: If there are enough studies, we will conduct subgroup analysis to explore the possible reasons for heterogeneity. Subgroup analysis will be carried out according to the modeling method and acupoint combination method.

Sensitivity analysis: We will conduct sensitivity analyses based on study quality.

Country(ies) involved: China.

Keywords: Acupuncture; Vascular dementia; animal; Meta analysis.

Contributions of each author:

Author 1 - Guangyao Li.
Author 2 - Yuling Shi.

Author 3 - Chuanghui Yang.

Author 4 - Jinghu Li.

Author 5 - Xueqin Hong.

Author 6 - Min Li. 